\title{
Time to follow guidelines, protocols, and structured procedures in medical care and time to leap out
}

This article was published in the following Dove Press journal:

Risk Management and Healthcare Policy

21 November 2014

Number of times this article has been viewed

\author{
Ayala Kobo-Greenhut ${ }^{1-3}$ \\ Amos Notea ${ }^{2,3}$ \\ Meir Ruach ${ }^{4}$ \\ Erez Onn ${ }^{4}$ \\ Yehunatan Hasin' \\ 'Bar-Ilan University, Tel Aviv, Israel; \\ ${ }^{2}$ Technion, Haifa, Israel; ${ }^{3}$ Kinneret \\ College, Galilee, Israel; ${ }^{4}$ Baruch Padeh \\ Medical Center, Poriya, Israel
}

\begin{abstract}
Present medical practice encourages management according to written guidelines, protocols, and structured procedures (GPPs). Daily medical practice includes instances in which "leaping" from one patient management routine to another is a must. We define "frozen patient management", when patient management leaping was required but was not performed. Frozen patient management may cause significant damage to patient safety and health and the treatment quality. This paper discusses the advantages and disadvantages of GPP-guided medical practice and gives an explanation of the problem of frozen patient management in light of quality engineering, control engineering, and learning processes. Our analysis of frozen patient management is based on consideration of medical care as a process. By considering medical care processes as a closed-loop control process, it is possible to explain why, when an indication for deviation from the expected occurs, it does not necessarily attract the medical teams' attention, thereby preventing the realization that leaping to an alternative patient management is needed. We suggest that working according to GPPs intensifies the frozen patient management problem since working according to GPPs relates to "exploitation learning behavior", while leaping to new patient management relates to "exploration learning behavior". We indicate practice routines to be incorporated into GPP-guided medical care, to reduce frozen patient management.
\end{abstract}

Keywords: guidelines, protocols, structured procedures, frozen patient management, close loop

\section{Introduction \\ One of many}

One of the court rulings on medical negligence in Israel was the subject of delayed diagnosis of retinal detachment, which resulted in severe visual loss in the patient. The patient was diagnosed with infectious conjunctivitis that deteriorated. The doctors (diagnosticians and those who provided care) were captive by the diagnosis and guidelines for infectious conjunctivitis and did not consider other reasons for the patient's visual deterioration. The end result was that they did not leap to the retina separation diagnosis and guideline until the damage became irreversible. When finally the retinal detachment was discovered, the visual loss was irreversible (case A51694/04).

The primary purpose of this paper is to introduce quality engineering terminology and techniques into medical practice. Specifically, we aimed to discuss the advantages and disadvantages of medical practice following written guidelines, protocols, and structured procedures (GPPs) and the problem of "frozen patient management (PM)" that inhibits timely "leaping" from one PM routine to another. The above scenario is an example of a medical team that continued with a selected PM, without replacing it with a more appropriate one (ie, leaping from one PM routine to another). While there
Correspondence: A Kobo-Greenhut Email ayala.kgl@gmail.com 
is no doubt about the benefits and importance of working according to written GPP, our approach is that there are cases where leaping from one PM routine to another is a must.

Present medical practice encourages management according to written GPPs. The GPPs are based on current evidence and experience, providing a sense of security, organization, and order to the work. In most instances, working according to GPPs results in the optimization of patient care. It also gives peace of mind to the caregiving team. Nevertheless there are cases where replacing the chosen GPP by an alternative one may be required (ie, leaping from one PM routine to another). For instance, leaping may be required when the initial PM was based on data of high uncertainty and was created before additional, clearer data or data validation was available, thus leading to choice of the wrong GPP. Moreover, even when the chosen GPP is correct, the specific patient may not react as expected to the treatment. This may happen since GPPs fit most of the patients with the specific problem but not all.

Despite the importance of timely leaping from a decided GPP to another, there are events when medical teams fail to do so. We define "frozen PM" as a failure to leap. Frozen PM may cause significant damage to patient safety, health, and the treatment quality.

The following discussion is an attempt to analyze the problem of frozen PM in light of quality engineering, control engineering, and learning processes that have been shown to improve function in industries. The adaptation of quality and engineering concepts to the health care domain started in the 1980s, with a main emphasis on the structural process and outcome aspects of medical care, by several researchers ${ }^{1-6}$ who shared the view that: "The current American healthcare system is in crisis ... engineering can be used to transform the healthcare system into one that is safe, effective, patient centered, timely, efficient, and equitable". 7 It is important to notice that attention was given to structural process and outcome aspects of medical care and not to the medical care professionals' decisions and processes. The following includes our suggestion to adapt the concepts of quality engineering, control engineering, and learning to medical care.

Our explanation for frozen PM is based on consideration of medical care as a process. By considering medical care processes as closed-loop control processes, it is possible to explain why, when an indication for deviation from expected occurs, it does not necessarily attract the medical teams' attention, thereby preventing the realization that leaping to an alternative PM is needed. We call these indications gaps - rifts between expected and actual output results.
Here, we adopt an approach based on analysis of closed-loop control systems ${ }^{8}$ demonstrating that when gaps occur, the controller compensates for it (ie, indicating "false alarm") and prevents its realization. Only extremely large gaps may really be realized as failures that require response.

We suggest that frozen PM occurs since PM is a closedloop process it suffers from the problem of not realizing gaps as failures. Although in principle, the problem seems to be similar, ie, not recognizing gaps as failures, the mechanisms to explain it are different. Frozen PM occurs when gaps are considered to be part of the current PM without raising explicit attention to anomalies - even if the actual state is, in fact, quite different. In the medical care closed-loop process, the "controller" is the human mind.

Moreover, working according to GPPs intensifies the frozen PM problem since working according to GPP relates to an "exploitation" learning behavior, while leaping to new PM relates to an "exploration" learning behavior. Traditionally, exploration and exploitation learning behaviors are related to research and development. We adapt these terms to patient care. Organizational learning is "the process of improving actions through better knowledge and understanding", 9,10 while learning behavior consists of activities through which data are obtained and processed. ${ }^{11}$ Exploitation is defined as the "ongoing use of a firm's knowledge base"; 12 therefore exploitative behavior emphasizes standardization, uniformity, low risk, consistency, and low variance. Exploration is defined as "search for new knowledge"; 12 therefore explorative behavior emphasizes discretion, high variability, and high risks.

We suggest that although GPPs emphasize exploitative behavior, they enfeeble exploration learning behavior. ${ }^{13,14}$ As a result, working according to GPPs intensifies the frozen PM problem.

\section{Doctors in a closed loop}

A closed-loop system is a system in which the controller is used to automatically modify the control input of a plant in such a way that the process remains stable, and the output remains as close as possible to its desired value. ${ }^{14}$ For example, for engineering a closed-loop system one could envision a heat exchanger that should heat water to a specific temperature. If the water temperature is lower than the target value, more heated steam is made to flow.

In order to explain unidentified failures in medical care, we modeled the medical care process as a closed-loop one (Figure 1), based on Wagner's logical model of medical diagnosis. $^{15}$ 
The process model contains the following blocks:

- Database: The teams evaluate $P\left(D_{i}\right)$, the probability that the patient has a particular set of diseases $D_{i}$

- Patient status: The teams evaluate $P\left(S_{j} \mid D_{i}\right)$, the probability of the occurrence of a syndrome $S_{j}$, given a particular set of diseases $D_{i}$

- Evaluation block: The medical team evaluates $P\left(D_{i} \mid S_{j}\right)$, the probability of the patient having the set of diseases $D_{i}$, expressed by the syndrome $S_{j}$; and $P\left(D_{i}^{\prime} \mid S_{j}\right)$, the probability that the patient does not have the set of diseases $D_{i}^{\prime}$, given the syndrome $S_{j}$. These probabilities are estimated by analyzing $P\left(D_{i}\right), P\left(S_{j} \mid D_{i}\right)$, and the patient manifestation $m_{i}$

- Diagnosis block: The medical team determines the patient diagnosis, derived from the evaluation block

- Treatment block: The medical team treats the patient according to the treatment plan. The patient manifestation $m_{i}$ is received from this block. The patient manifestations at $t=0$ are the initial ones.

As time progresses, the manifestations change with the treatment, as is the patient's response to it. The patient's manifestation $m_{i}$ returns to the evaluation block as an input to the decision of whether the PM should remain unchanged and stay in the closed-loop or leap to another PM (different closed-loop). When frozen PM occurs, ie, when the manifestations $m_{i}$ deviate from the expected values $\hat{m}_{i}$, it should not necessarily be considered as relating to failure.

Relating to the heat exchanger example, if a hole occurs in one of the steam pipes, additional steam is made to flow, and the output water temperature is kept at the temperature target value. When monitoring the water temperature, it is not identified as a clear deviation from the temperature target unless several deviations occur; the reason is that the controller has compensated by increasing the released steam. Similarly, the

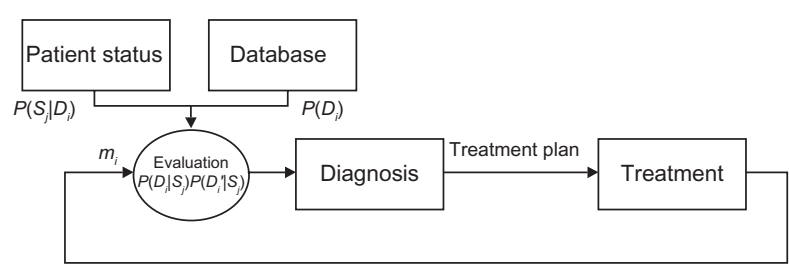

Figure I Medical care process modeled as a closed-loop process.

Notes: $P\left(D_{i}\right)$ is the probability that the patient has a particular set of diseases $D_{i}$. $P\left(S_{j} \mid D_{i}\right)$ is the probability of the occurrence of a syndrome $S$, given a particular set of diseases $D_{i} \cdot P\left(D_{i} \mid S_{j}\right)$ is the probability of the patient having the set diseases $D_{i}$, expressed by the syndrome $S_{j} P\left(D_{i}^{\prime} \mid S_{j}\right)$ is the probability that the patient does not have the set of diseases $D_{i}^{\prime}$, given the syndrome $S_{j} . m_{i}$ is the patient manifestation. medical team may relate to a deviated manifestation (ie, gap) as resulting from other reasons, such as technical problems (equipment mishaps) or delay in the patient's response to treatment. Only when the gap is extremely large or the medical manifestations are very unexpected and unambiguous, will the team reconsider the gap as a failure.

In medical care processes, the "controller" is the human mind, which does not identify the gaps as failures, mainly due to factors such as biases and habitual routine. Anchoring bias is the common human tendency to rely too heavily, or "anchor", on one trait or piece of information when deciding on the PM, locking on to salient features in a PM too early and failing to adjust in light of later information. ${ }^{16}$ Furthermore, the wrong PM can even drive the medical team to look for tests that will support its PM rather than searching for additional options, creating the "confirmation bias": Looking for evidence to support a PM rather than looking for evidence that might rebut it. " ${ }^{17}$ "Ego bias is manifested in the warping of probability estimates in a self-serving way". ${ }^{18}$ Recent study has shown that both individual physicians and medical teams show great confidence in their judgments, even when it can be shown to be incorrect. ${ }^{18}$ A physician's ego is affected by patients, who often need to consider their physician as someone who never fails, especially in critical situations involving a patient's life. Since physicians often believe that they will beat the overall odds of success for any venture in which they participate ${ }^{18}$ and that they are always right, they fail to ask for a second opinion, even in risky cases. Medical teams may not change the PM since this can be perceived as admitting a failure to predict the correct PM.

"A habitual routine exists when a group repeatedly exhibits a functionally similar pattern of behavior in a given stimulus situation without explicitly selecting it over alternative ways of behaving". ${ }^{19,20}$ We claim that frozen PM is a specific aspect of habitual routine. It relates to a medical team that is not aware of the need to leap to an alternative PM, even when the results indicate that the current PM is inappropriate and irrelevant. Teams, like individuals, often develop habitual routines for dealing with frequently encountered stimuli. Habitual routine drives teams to ignore alternative behavior since automatically, new or different data are considered to comply with the same PM, without giving explicit attention to anomalies. ${ }^{19,20}$ This phenomenon is very prevalent in medical teams since habitual routine often occurs when dealing with a process subjected to frequently changing stimuli, as happens in medical care. 


\section{Frozen PM, GPPs, and learning} GPPs, in which organizations store knowledge, ${ }^{21}$ emphasize controlling the variance in processes and outcomes. ${ }^{22}$ They reduce the variance and the personnel's ambiguity, ${ }^{23}$ and as a result, emphasize low-variance establishment and ensure uniform execution. GPPs are based on knowledge and experience, are considered fit, and the general advice they proffer is thought to be applicable to a broad range of cases. ${ }^{24}$ Working according to GPPs relates to exploitation learning behavior.

While GPPs relate to exploitation learning behavior, which emphasizes standardization, uniformity, low risk, consistency, and low variance, leaping to an alternative PM requires discretion, high variability, flexibility, experimentation, and divergent thinking. Leaping to an alternative PM is largely dependent on the ability of the medical team to be continuously aware of the importance of updating and expanding knowledge and data in light of changing data and environmental conditions, as well as the essentiality of PM changing with time. This can and should use internal as well as external options and ideas. It means that the medical team cannot afford to rely entirely on their "inside" subdivision knowledge; they should also look for "outside" options. In patient care, the treating medical teams are usually experts in one discipline, with a limited set of information, and thus are bound to a lower level of flexibility. The medical team should be open to incorporating an outsider's input. Leaping to an alternative PM requires exploration learning behavior. Exploration learning behavior consists of activities such as searching for, discovering, creating, and experimenting with new opportunities; ${ }^{20}$ experimenting with new approaches ${ }^{25}$ innovating, ${ }^{25-28}$ and reconsidering existing beliefs and decisions. ${ }^{29-31}$

GPPs restrict exploration learning behavior since their structured nature makes adaptation difficult. ${ }^{14}$ The variationreducing focus restricts the development of alternatives, ${ }^{13}$ hampers the discretion of the employees, ${ }^{32}$ leads to resistance to change and momentum, and thus, inhibits variability, ${ }^{13}$ thereby leading to an "easy road" of habitual routines. Moreover, welllearned guidelines and procedures generally contribute to individual members' comfort with the department - well-learned guidelines and procedures increase members' confidence about their roles in the department, and reduce the risk of "doing the wrong thing" and being perceived as a deviant.

\section{Integration of exploitation and exploration learned behavior applied to medical practice}

Two approaches have been introduced to provide explanations of how organizations can achieve both exploration and exploitation learning behaviors. The first approach, "ambidextrous", refers to highly differentiated but weakly integrated subunits. ${ }^{30}$ According to this approach, organizational units should specialize in either exploration or exploitation learning behaviors. Exploration units should be located in different places, have different personnel, and even be related to suborganizations that are distinct from the "mother firm". ${ }^{13}$ In medicine, this may be analogous to different functions characterizing academic and nonacademic institutions. The second approach, "punctuated equilibrium", refers to temporal rather than organizational structure differentiation. It suggests organizational transformation through cycles of long time periods of exploitation learning behavior and short bursts of exploration learning behavior. ${ }^{33}$ This approach enables organizations to balance between exploration and exploitation learning behaviors as they shift from one activity to another. ${ }^{34}$ This approach is difficult to apply to medical practice, which needs an overall continuous, uninterrupted practice routine. Yet perhaps focal, pointed organizational leaping may occur.

Unfortunately, medicine requires a new approach that combines the two accepted and contradictory approaches, both of which deal with the balance between exploration and exploitation learning behaviors. On the one hand, in line with the ambidextrous approach, we posit that exploitation and exploration learning behaviors are separate and derive from the PM leaping. On the other hand, since the interplay is generally short (in terms of time) and dynamic, it can be said that the two learning behaviors exist simultaneously, as in the punctuated equilibrium approach. Moreover, while the existence of interplay has been discussed in several studies, ${ }^{33,35-37}$ other authors believe that interplay occurs over a relatively long period of time. We, on the other hand, suggest that the interplay (ie, leaping) can occur over very short time periods: days or even hours. In order to reduce frozen PM occurrence, daily routine clinical practice should be able to incorporate exploration into its routine exploitation infrastructure. Further research is still needed before appropriate guidelines can be written on the subject.

\section{Conclusion}

In most cases, working in closed-loop systems according to GPPs results in the optimization of patient care and maximization of treatment quality and safety. In some cases, leaping from one PM to another may be required. Despite the importance and benefits of the introduction of GPP into the PM routine, some disadvantages will be incorporated. Working according to GPPs intensifies the problem of 
frozen PM. Consequently, working according to GPPs must be established with a methodology that will ensure PM leaping when required. Further research is needed to achieve the goal of introducing structured integration of exploitation and exploration learning behavior into medical practice.

\section{Disclosure}

The authors report no conflicts of interest in this work.

\section{References}

1. Donabedian A. An Introduction to Quality Assurance in Health Care. Oxford: Oxford University Press; 2002.

2. Carey RG, Lloyd RC. Measuring Quality Improvement in Healthcare: A Guide to Statistical Process Control Applications. Milwaukee, WI: American Society for Quality; 2001.

3. Nemeth C, O'Connor M, Klock PA, Cook R. Discovering healthcare cognition: the use of cognitive artifacts to reveal cognitive work. Organ Stud. 2006;27(7):1011-1035.

4. Lynn J, Baily MA, Bottrell M, et al. The ethics of using quality improvement methods in health care. Ann Intern Med. 2007;146(9): 666-673.

5. Nelson EC, Batalden PB. Patient-based quality measurement systems. Qual Manag Health Care. 1993;2(1):18-30.

6. Gardner G, Gardner A, O'Connell J. Using the Donabedian framework to examine the quality and safety of nursing service innovation. $J$ Clin Nurs. 2014;23(1-2):145-155.

7. Green M, Nembhard HB. Literature Review of Healthcare Delivery. State College, PA: Penn State University; 2009. Available from: http:// www.cihds.psu.edu/pdf/literature $\% 20$ review $\% 20$ of $\% 20$ healthcare $\% 20$ delivery.pdf. Accessed October 13, 2014.

8. Kobo-Greenhut A. Integration of Feedback Control and Statistical Process Control [master's thesis]. Haifa: Technion; 2009.

9. Fiol MC, Lyles MA. Organizational learning. Acad Manage Rev. 1985;10:803-810.

10. Argote L. Organization Learning: Creating, Retaining and Transferring Knowledge. New York, NY: Springer; 2013.

11. Edmondson A. Psychological safety and learning behavior in work teams. Admin Sci Quart. 1999;44(2):350-383.

12. Vermeulen F, Barkema H. Learning through acquisition. Acad Manage J. 2001;44(3):457-478.

13. Benner MJ, Tushman ML. Exploitation, exploration, and process management: The productivity dilemma revisited. Acad Manage Rev. 2003;28(2):238-256.

14. Brown SL, Eisenhardt KM. Competing on the Edge - Strategy as Structured Chaos. Boston, MA: Harvard Business Press; 1998.

15. Wagner HN Jr. Regional ventilation and perfusion. In: Wagner HN Jr, Szabo Z, Buchanan JW, editors. Principles of Nuclear Medicine. Philadelphia, PA: Saunders; 1995:881-895.

16. Croskerry $\mathrm{P}$. The importance of cognitive errors in diagnosis and strategies to minimize them. Acad Med. 2003;78(8):775-780.

17. Ogata K. Modern Control Engineering. 3rd ed. Upper Saddle River, NJ: Prentice Hall; 1996.
18. Dawson NV, Arkes HR. Systematic errors in medical decision making: judgment limitations. J Gen Intern Med. 1987;2(3):183-187.

19. Gersick CJ, Hackman JR. Habitual routines in task-performing groups. Organ Behav Hum Decis Process. 1990;47:65-97.

20. Day RD. Leading and Managing People in the Dynamic Organization. Psychology Press, 2014.

21. March JG. Exploration and exploitation in organizational learning. Organ Sci. 1991;2(1):71-87.

22. Juran JM, Godfrey AB. Juran's Quality Handbook. 5th ed. New York, NY: McGraw-Hill; 1998.

23. Tatikonda MV, Montoya-Weiss MM. Integrating operations and marketing perspectives of product innovation: The influence of organizational process factors and capabilities on development performance. Manage Sci. 2001;47(1):151-172.

24. Matusik SF, Hill CWL. The utilization of contingent work, knowledge creation, and competitive advantage. Acad Manage Rev. 1998;23(4): 680-697.

25. McGrath RG. Exploratory learning, innovative capacity, and managerial oversight. Acad Manage J. 2001;44(1):118-131.

26. Duncan RB. Modifications in decision structure in adapting to the environment: Some implications for organizational learning. Decision Sci. 1974;5(4):705-725.

27. Martini A, Gastaldi L, Corso M, Laugen BT. Continuous innovation: towards a paradoxical, ambidextrous combination of exploration and exploitation. Int J Technol Manage. 2013;61(1):1-22.

28. Tushman M, O'Reilly C. Ambidextrous organizations: managing evolutionary and revolutionary change. Calif Manage Rev. 1996;38(4): $8-30$.

29. Floyd SW, Lane PJ. Strategizing throughout the organization: Managing role conflict in strategic renewal. Acad Manage Rev. 2000;25(1): 154-177.

30. Ghemawat P, Ricart Costa JEI. The organizational tension between static and dynamic efficiency. Strategic Manage J. 1993;14(S2):S59-S73.

31. Rivkin JW, Siggelkow N. Balancing search and stability: Interdependencies among elements of organizational design. Manage Sci. 2003;49(3):290-311.

32. Cropanzano R, Byrne ZS, Bobocel DR, Rupp DE. Moral virtues, fairness heuristics, social entities, and other denizens of organizationalv justice. J Vocat Behav. 2001;58(2):164-209.

33. Gupta AK, Smith KG, Shalley CE. The interplay between exploration and exploitation. Acad Manage J. 2006;49(4):693-706.

34. Romanelli E, Tushman ML. Organizational transformation as punctuated equilibrium: An empirical test. Acad Manage J. 1994;37(5): 1141-1166.

35. Lewis MW, Walsh MA, Dehler GE, Green SG. Product development tensions: exploring contrasting styles of project management. Acad Manage J. 2002;45(3):546-564.

36. Raisch S, Birkinshaw J, Probst G, Tushman ML. Organizational ambidexterity: balancing exploitation and exploration for sustained performance. Organ Sci. 2009;20(4):685-695.

37. Hjelmgren D, Dubois A. Organising the interplay between exploitation and exploration: The case of interactive development of an information system. Ind Market Manag. 2013;42(1):96-105.
Risk Management and Healthcare Policy

\section{Publish your work in this journal}

Risk Management and Healthcare Policy is an international, peerreviewed, open access journal focusing on all aspects of public health, policy, and preventative measures to promote good health and improve morbidity and mortality in the population. The journal welcomes submitted papers covering original research, basic science, clinical \& epidemio-

\section{Dovepress}

logical studies, reviews and evaluations, guidelines, expert opinion and commentary, case reports and extended reports. The manuscript management system is completely online and includes a very quick and fair peerreview system, which is all easy to use. Visit http://www.dovepress.com/ testimonials.php to read real quotes from published authors 